\title{
Distance Extraction by Means of Photon-Counting Passive Sensing Combined with Integral Imaging
}

\author{
Seokwon Yeom*, Yong-Hyen Woo, and Won-Woo Baek \\ Division of Computer and Communication Engineering, Daegu University, \\ Gyeongsan 712-714, Korea
}

(Received August 25, 2011 : revised November 15, 2011 : accepted December 8, 2011)

\begin{abstract}
Photon-counting sensing is a widely used technique for low-light-level imaging applications. This paper proposes a distance information extraction method with photon-counting passive sensing under low-lightlevel conditions. The photo-counting passive sensing combined with integral imaging generates a photon-limited elemental image array. Maximum-likelihood estimation (MLE) is used to reconstruct the photon-limited image at certain depth levels. The distance information is extracted at the depth level that minimizes the sum of the standard deviation of the corresponding photo-events in the elemental image array. Experimental and simulation results confirm that the proposed method can extract the distance information of the object under low-light-level conditions.
\end{abstract}

Keywords : Distance extraction, Integral imaging, Image reconstruction, Photon-counting, Passive sensing OCIS codes : (100.0100) Image processing; (110.6880) Three-dimensional image acquisition; (150.0150) Machine vision; (030.5260) Photon counting; (040.3780) Low light level

\section{INTRODUCTION}

Distance information extraction has been the subject of research for numerous applications [1-3]. For depth information to be extracted, three-dimensional (3D) information needs to be acquired and processed. For example, stereo images or a sequence of images are often matched to each other to extract depth information based on pixel disparity $[2,3]$.

Integral imaging (II) is primarily a 3D display technique but it has been widely adopted for information processing such as obtaining depth information and object recognition [4-11]. During II recording, an elemental image array is generated, that has different views of the object. One advantage of II is that only a single exposure is required to obtain 3D information; no calibration is needed, unlike stereo imaging, and no active illumination is needed, unlike holography or light detection and ranging (LIDAR) $[12,13]$. A depth extraction technique using elemental images has been studied in [8-11]. Depth is extracted by means of one-dimensional elemental image modification and a correlation-based multi-baseline stereo algorithm [10]. In [11], the depth level of the reconstruction plan is estimated by minimizing the sum of the standard deviations of the corresponding pixels' intensity.

Photon-counting imaging has been developed for lowlight-level imaging applications such as night vision, and laser radar, radiological, and stellar imaging [14-18]. Advanced photon-counting imaging technology can register a single photo-event at each pixel. In that case, photo-detection is carried out in the binary mode generating a binary dotted image. The object recognition with nonlinear matched filtering is proposed in [19]. II reconstruction with maximum likelihood estimation (MLE) is proposed in [20]. Stereoscopic photoncounting sensing has been proposed for distance information extraction [21].

This paper proposes the use of photon-counting passive sensing combined with integral imaging for distance information extraction under low-light-level conditions. Photonlimited imagery is reconstructed with maximum likelihood estimation (MLE). In this paper, the MLE for photon-limited scene reconstruction in 3D space is proposed with the Poisson distribution in [20], while the probability model is modified according to the low-light-level conditions. It has been shown that the MLE is merely the average of the photo-events in the elemental image array. Those photoevents are associated with pixels corresponding to a specific

\footnotetext{
*Corresponding author: yeom@daegu.ac.kr

Color versions of one or more of the figures in this paper are available online.
} 
point in 3D space. The obtained depth level is the distance that minimizes the sum of the standard deviations of the corresponding photo-counts. The sum of the standard deviations represents the uncertainty of the sampled information. There have been efforts to minimize the uncertainty in order to reconstruct the occluded scene in [1] and the intensity elemental image array in [11].

Photon-limited elemental images are simulated on a computer while varying the expected total number of photo-events. The performance is evaluated accordingly. We also compare the distance extraction between photon-limited and intensity elemental images. The uncertainty minimization has been applied to both photo-event and intensity cases and consistent results are obtained from both cases. The experimental results confirm that the proposed method can extract distance information under low-light-level conditions. To the best of the authors' knowledge, it is the first report on distance extraction by use of photon-counting passive sensing combined with integral imaging.

The rest of the paper is organized as follows. Section 2 describes the photo-event model and the distance information extraction algorithm. The experimental and simulation results are presented in Section 3. Conclusion follows in Section 4.

\section{DISTANCE INFORMATION EXTRACTION WITH PHOTON-COUNTING INTEGRAL IMAGING}

The II recording system generates an elemental image array as illustrated in Fig. 1. The microlens array is composed of a large number of small convex lenslets, and the ray information captured by each lenslet appears as an elemental image, that has different view of the object.

Under low-light-level conditions, a photo-detector can register a single photo-event and generate a binary dotted elemental image array. It can be assumed that the probability of a photo-event is proportional to the intensity of the pixel at a low-light level [15]. Thus, the following probability model is valid:

$$
P_{d}\left(y_{i}=1 ; n_{i}\right)=n_{i}, \quad P_{d}\left(y_{i}=0 ; n_{i}\right)=1-n_{i},
$$

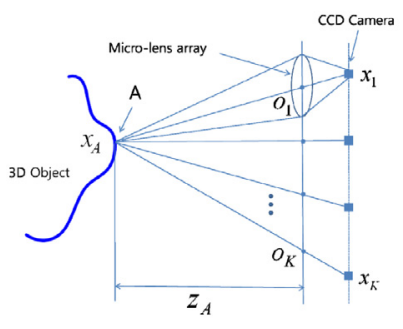

(a)

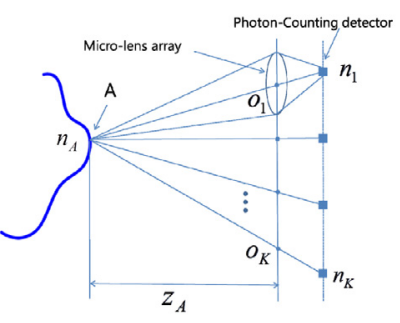

(b)
FIG. 1. Integral imaging system with, (a) CCD camera, (b) photo-detector.

$$
n_{i}=N_{p} x_{i}
$$

where $y_{i}$ indicates a single photo-event at pixel $i, N_{p}$ is an expected total photo-counts in the scene, $x_{i}$ is the normalized intensity at pixel $i$, i.e., $\sum_{i=1}^{N_{t}} x_{i}=1$, where $N_{t}$ is the total number of pixels in the scene. It can be seen that $E\left(y_{i}\right)=$ $n_{i}$ and $E\left(\sum_{i=1}^{N_{t}} y_{i}\right)=N_{p}$.

Let $y_{i}$, where $i=1,2, \cdots, K$, is the number of photocounts detected at pixel $i$, which corresponds to the $x_{i} ; K$ is the number of lenslets, which captures the point $A$ in Fig. 1. The joint probability distribution of $y_{1}, \cdots, y_{K}$, since they are independently registered, becomes

$$
P_{d}\left(y_{1}, \ldots, y_{K} ; n_{1}, \ldots, n_{K}\right)=n_{1}^{y_{1}} \cdots n_{K}^{y_{K}}\left(1-n_{1}\right)^{\left(1-y_{1}\right)} \cdots\left(1-n_{K}\right)^{\left(1-y_{K}\right)} \text {. }
$$

It can be assumed that all $n_{i}$ 's are equal and proportional to intensity $n_{A}$ at the point $A$ in the reconstruction plane as illustrated in Fig. 1(b), thus Eq. (3) simplifies to

$$
P_{d}\left(y_{1}, \ldots, y_{K} ; n_{A}\right)=n_{A}^{\sum_{i=1}^{K} y_{i}}\left(1-n_{A}\right)^{K-\sum_{i=1}^{K} y_{i}},
$$

The MLE (maximum likelihood estimation) of $n_{A}$ in Eq. (4) is obtained as

$$
\hat{n}_{A}=\frac{1}{K} \sum_{i=1}^{K} y_{i},
$$

which is the average of the photo-counts originated from the point $A$.

The sum of the standard deviation of the photo-counts over the reconstruction plane is chosen for our metric. It is assumed that the distance level minimizes the sum of standard deviations of the corresponding photo-counts. Therefore, the depth $z$ to the reconstruction plane is estimated as

$$
\begin{aligned}
& \hat{z}=\min _{z} \sum_{j=1}^{N_{r}} s_{j}(z) \\
& s_{j}^{2}(z)=\frac{1}{K_{j}} \sum_{i=1}^{K_{j}}\left(y_{j, i}-\hat{n}_{j}\right)^{2}, \\
& \hat{n}_{j}=\frac{1}{K_{j}} \sum_{i=1}^{K_{j}} y_{j, i},
\end{aligned}
$$

where $N_{r}$ is the number of voxels in the reconstruction plane, $K_{j}$ is the number of the lenslets capturing the voxel 
$j$, and $y_{j, i}$ is the photo-counts in the imaging plane associated with the voxel $j$.

Eqs. (6) and (8) are equivalent with Eqs. (9) and (11), which extract distance information in the conventional intensity elemental images as the following [11]:

$$
\begin{aligned}
& \hat{z}_{v}=\min _{z} \sum_{j=1}^{N_{r}} \sigma_{j}(z) \\
& \sigma_{j}^{2}(z)=\frac{1}{K_{j}} \sum_{i=1}^{K_{j}}\left(x_{j, i}-\hat{x}_{j}\right)^{2}
\end{aligned}
$$

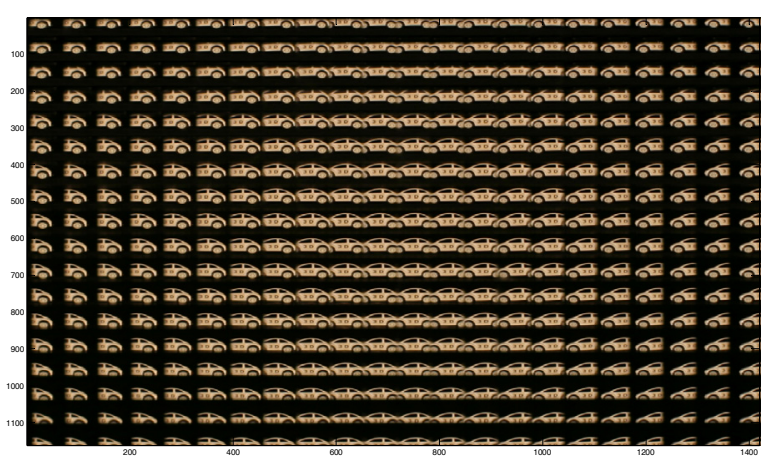

FIG. 2. An elemental image array.

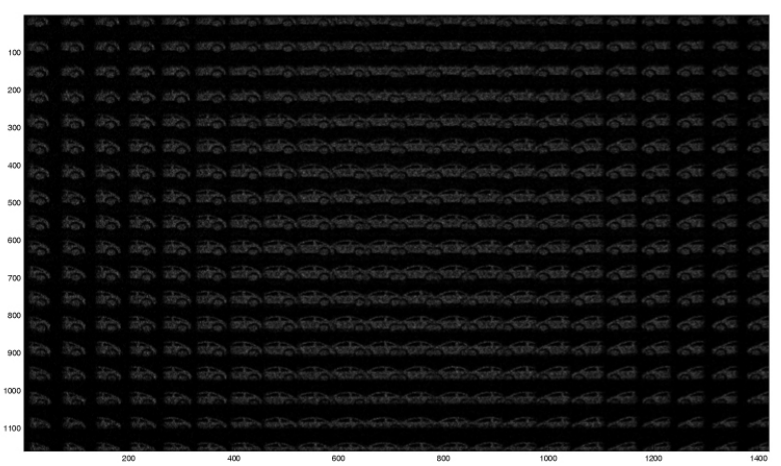

(a)

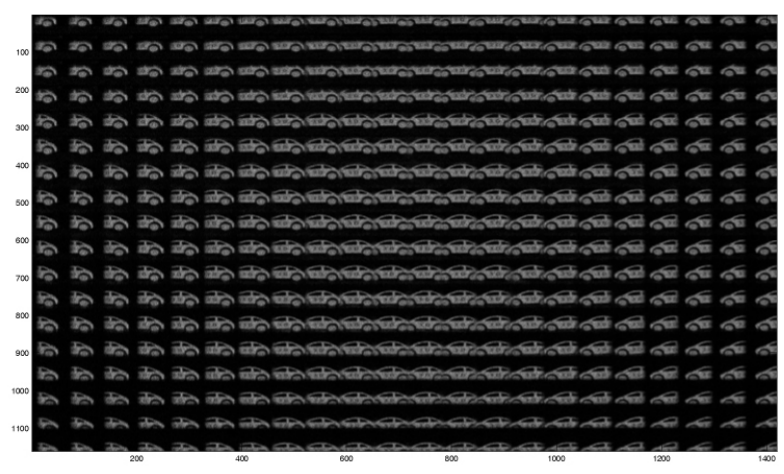

(c)

$$
\hat{x}_{j}=\frac{1}{K_{j}} \sum_{i=1}^{K_{j}} x_{j, i},
$$

In the next section, we evaluate the performance of the depth extraction using Eq. (6) with different expected total number of photo-events. The distance extraction derived by intensity images in Eq. (9) is also compared with the photon-limited images.

\section{EXPERIMENTAL RESULTS}

The II recording system is composed of a microlens array and a pick-up camera. The pitch of each lenslet is $1.09 \mathrm{~mm}$, and the focal length of each lenslet is about 3.3 $\mathrm{mm}$. One toy car is used in the experiments. Figure 2 shows the elemental image array. The size of the elemental image array is $1419 \times 1161$ pixels and the number of elemental images is $22 \times 18$. One-hundred photon-limited elemental image arrays are generated using a pseudo random number generator on a computer. Figures 3(a)-(d) show the examples of the photon-limited images while varying photo-counts; $N_{p}$ varies as $1 \times 10^{6}, 5 \times 10^{6}, 1 \times 10^{7}$, and $5 \times 10^{7}$.

Figures 4 is a plot of the sum of the standard deviations obtained from a gray-scaled image according to Eq. (9) [11]. The sum of the standard deviations is minimized at

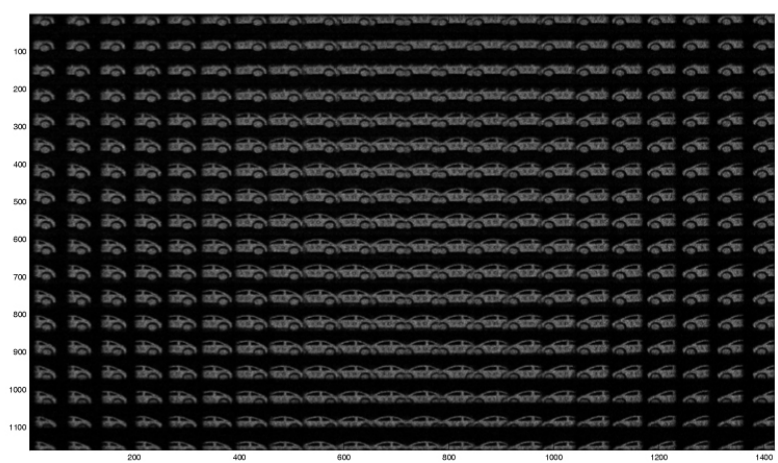

(b)

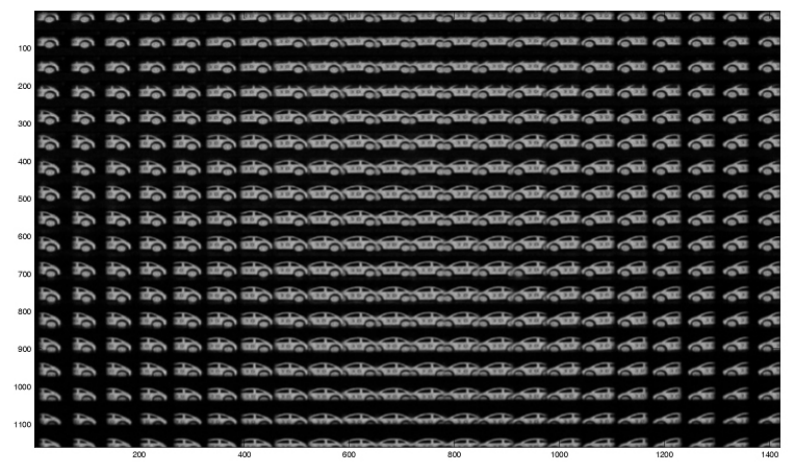

(d)

FIG. 3. Photon-limited elemental image arrays when $N_{p}$ is (a) $1 \times 10^{6}$, (b) $5 \times 10^{6}$, (c) $1 \times 10^{7}$, (d) $5 \times 10^{7}$. 
the depth level of $84 \mathrm{~mm}$. Figures 5(a)-(d) display the average and the error-bar of the standard deviations'sum

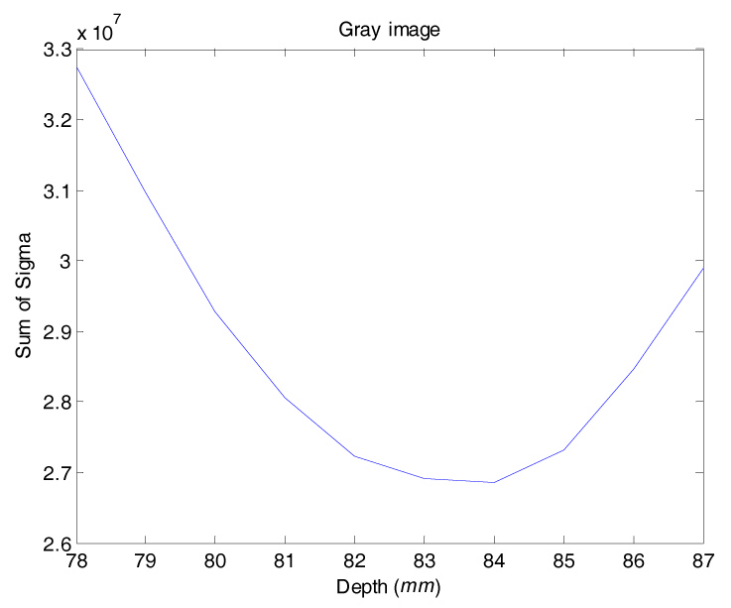

FIG. 4. Sum of standard deviations (SSD) with a gray scaled elemental image array.

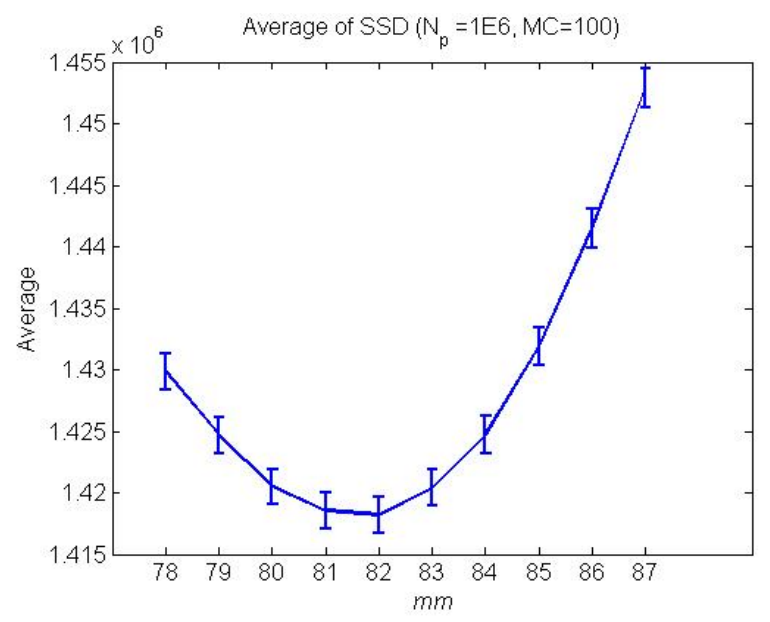

(a)

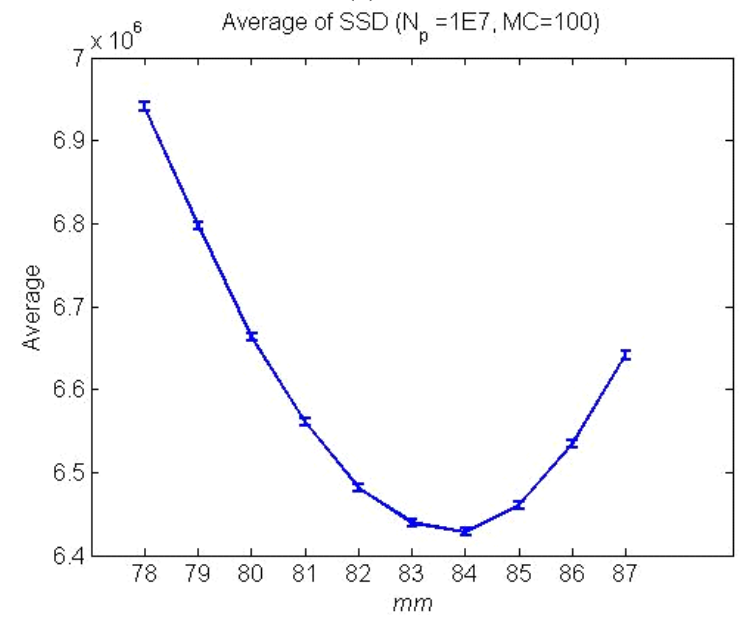

(c) over 100 photon limited images, which are obtained by Eq. (6). As more photo-events are acquired, the photonlimited image starts to resemble the intensity image in Fig. 3 , and the results in Fig. 5 approach those of Fig. 4. In this experiment, the depth level can be extracted when the photo-counts exceed $5 \times 10^{6}$ as illustrated in Fig. 5(b).

\section{CONCLUSIONS}

In this paper, a photon-counting integral imaging method for distance information extraction is proposed. The depth level of the object is determined by the distance at which the sum of the standard deviations of the photo-counts is minimized. The method is based on a compact system that requires only a single exposure under passive mode to obtain 3D information. Experimental and simulation results confirm that the proposed method can be used to obtain the distance information to an object at a low-light level. It was confirmed that the depth level is the same as the intensity images. Further investigation on the distance infor-

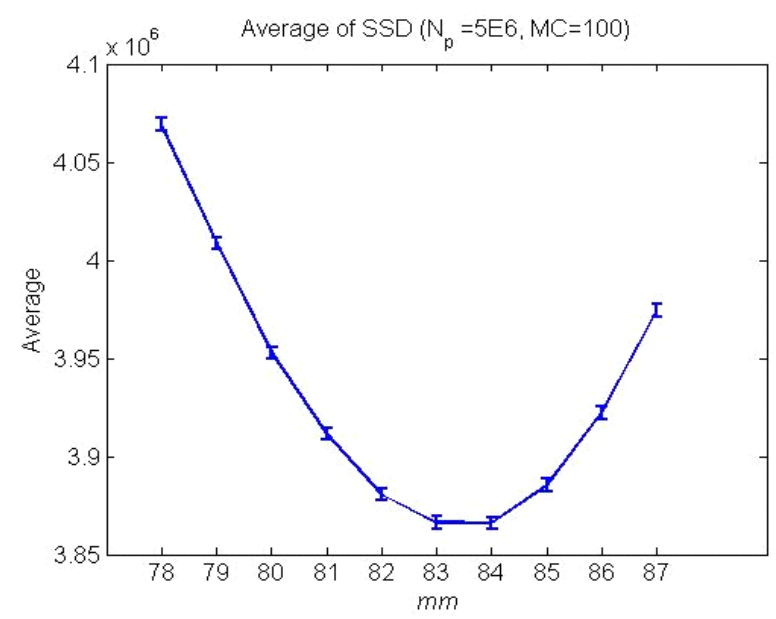

(b)

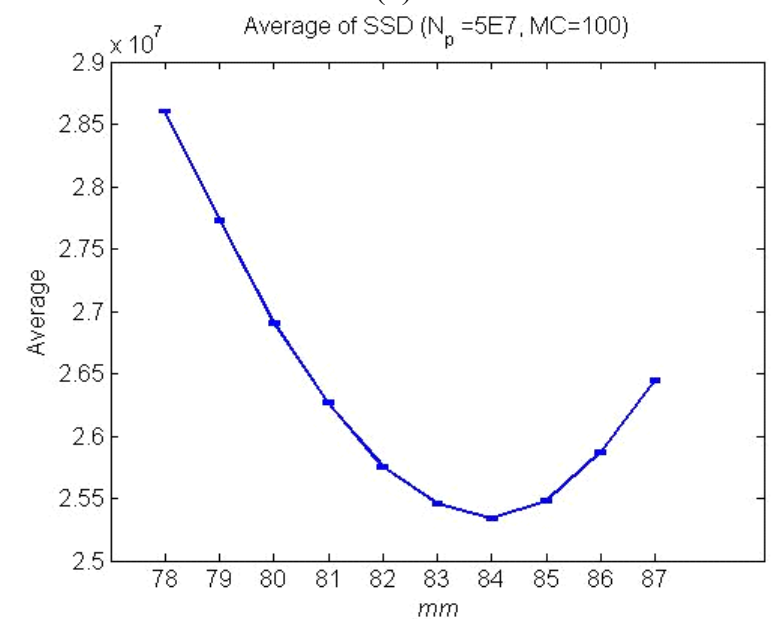

(d)

FIG. 5. Average and error bar of SSD over 100 photon-limited images when $N_{p}$ is (a) $1 \times 10^{6}$, (b) $5 \times 10^{6}$, (c) $1 \times 10^{7}$, (d) $5 \times 10^{7}$. 
mation extraction of multiple or occluded objects under low-light-level conditions remains for future study.

\section{ACKNOWLEDGMENT}

This research was supported by Basic Science Research Program through the National Research Foundation of Korea (NRF) funded by the Ministry of Education, Science, and Technology (No. 2011-0003853).

\section{REFERENCES}

1. Y. Y. Schechner and N. Kiryati, "Depth from defocus vs. stereo: how different really are they?," Proc. International Conference on Pattern Recognition, 1784-1786 (1998).

2. S.-W. Lee and N. Kim, "A method for precise depth detection in stereoscopic display," J. Opt. Soc. Korea 10, 37-41 (2006).

3. A. K. Dalmia and M. Trivedi, "Depth extraction using a single moving camera: an integration of depth from motion and depth from stereo," Machine Vision and Applications 9, 43-55 (1996).

4. G. Lippmann, "La photographie integrale," C. R. Acad. Sci. 146, 446-451 (1908).

5. J.-Y. Son, V. V. Saveljev, Y.-J Choi, J.-E. Bahn, S.-K Kim, and H. Choi, "Parameters for designing autostereoscopic imaging systems based on lenticular, parallax barrier, and integral photography plates," Opt. Eng. 42, 3326-3333 (2003).

6. S.-G. Park, B.-S. Song, and S.-W. Min, "Analysis of image visibility in projection-type integral imaging system without diffuser," J. Opt. Soc. Korea 14, 121-126 (2010).

7. J.-H. Park, J. Kim, and B. Lee, "Three-dimensional optical correlator using a sub-image array," Opt. Express 13, 5116-5126 (2005).

8. D.-C. Hwang, D.-H. Shin, S.-C. Kim, and E.-S. Kim, "Depth extraction of three-dimensional objects in space by the computational integral imaging reconstruction technique," Appl. Opt. 47, D128-D135 (2008).
9. C. Wu, M. McCormick, A. Aggoun, and S. Y. Kung, "Depth mapping of integral images through viewpoint image extraction with a hybrid disparity analysis algorithm," Journal of Display Technology 4, 101-108 (2008).

10. J.-H. Park, S. Jung, H. Choi, Y. Kim, and B. Lee, "Depth extraction by use of a rectangular lens array and one-dimensional elemental image modification," Appl. Opt. 43, 4882-4895 (2004).

11. D. Lee, S. Yeom, S. Kim, and J.-Y. Son, "Occluded object reconstruction and recognition with computational integral imaging," Hankook Kwanghak Hoeji (Korean J. Opt. Photon.) 19, 270-274 (2008).

12. N. T. Shaked, B. Katz, and J. Rosen, "Review of three-dimensional holographic imaging by multiple-viewpointprojection based methods," Appl. Opt. 48, H120-H136 (2009).

13. J. J. Degnan, "Photon counting lidars for airborne and spaceborne topographic mapping," in Proc. Applications of Lasers for Sensing and Free Space Communications (LSC) (San Diego, CA, USA, Feb. 2010), LSWA4.

14. E. Hecht, Optics, 4th ed. (Addison Wesley, San Francisco, USA, 2001).

15. J. W. Goodman, Statistical Optics (Johnwiley \& Sons, Inc., Boston, USA, 1985).

16. Ph. Refregier, F. Goudail, and G. Delyon, "Photon noise effect on detection in coherent active images," Opt. Lett. 29, 162-164 (2004).

17. G. M. Morris, "Scene matching using photon-limited images," J. Opt. Soc. Am. A 1, 482-488 (1984).

18. E. A. Watson and G. M. Morris, "Comparison of infrared upconversion methods for photon-limited imaging," J. Appl. Phys. 67, 6075-6084 (1990).

19. S. Yeom, B. Javidi, and E. Watson, "Photon counting passive 3D image sensing for automatic target recognition," Opt. Express 13, 9310-9330 (2005).

20. S. Yeom, B. Javidi, C.-W. Lee, and E. Watson, "Photoncounting passive 3D image sensing for reconstruction and recognition of partially occluded objects," Opt. Express 15, 16189-16195 (2007).

21. S. Yeom, "Stereoscopic photon counting passive sensing for extraction of distance information," 3D Res. 02, 03003 (2011). 